Vol. 9, nº 12005

Varia

\title{
L'État au quotidien : histoire de la Volkspolizei
}

Fabien Jobard

\section{OpenEdition}

Journals

Édition électronique

URL : https://journals.openedition.org/chs/389

DOI : $10.4000 /$ chs.389

ISSN : 1663-4837

Éditeur

Librairie Droz

\section{Édition imprimée}

Date de publication : 1 juillet 2005

Pagination : 139-144

ISBN : 978-2-600-01014-6

ISSN : 1422-0857

Référence électronique

Fabien Jobard, "L'État au quotidien : histoire de la Volkspolizei », Crime, Histoire \& Sociétés / Crime, History \& Societies [En ligne], Vol. 9, n¹ | 2005, mis en ligne le 16 février 2009, consulté le 25 mars 2022. URL : http://journals.openedition.org/chs/389 ; DOI : https://doi.org/10.4000/chs.389

Ce document a été généré automatiquement le 25 mars 2022

(C) Droz 


\title{
L'État au quotidien : histoire de la Volkspolizei
}

\author{
Fabien Jobard
}

\section{RÉFÉRENCE}

Thomas Lindenberger, Volkspolizei. Herrschaftspraxis und öffentliche Ordnung im SED-Staat. 1952-68. Cologne : Böhlau, coll. « Zeithistorische Studien », n²3, 2003, 504 pages.

1 L'ouvrage de Thomas Lindenberger porte sur la « Police populaire» (Volkspolizei) de la RDA « affermie », du socialisme sur le sol allemand à la prise de pouvoir par Honecker et les techniciens, en somme de 1952 à 1968. On pouvait craindre de la publication intégrale (plus de 500 pages d'un volume de grand format) de cette thèse d'habilitation qu'elle se résumât à une grande somme historique sur un objet d'histoire, la Volkspolizei. Loin d'être, ou plutôt loin d'être seulement, une remarquable somme de connaissances sur la Volkspolizei et ses Volkspolizisten, l'ouvrage de Thomas Lindenberger s'affirme comme une recherche fondamentale sur les modes d'exercice de la contrainte étatique, sur la différenciation politique ou encore sur la gouvernementalité.

2 Après avoir rappelé les étapes majeures de la constitution de la Volkspolizei entre 1945 et 1952 (chap. 1), Lindenberger montre la gémellité incomplète de cet organe et de ses prédécesseurs soviétiques (chap. 2) ainsi que le rôle directeur du Parti dans son fonctionnement interne (chap. 3) et démontre, à l'appui d'une très rigoureuse analyse $\mathrm{du}$ « processus législatif » ayant abouti après plus d'une décennie de querelles à la « Loi sur la Volkspolizei » (Volkspolizeigesetz - chap. 4), combien cette police fut toujours une institution fragile, partagée, précaire. Certes, des fondations pérennes étaient assurées à la VP, par la prégnance du modèle soviétique de société militarisée et l'importance du maintien de l'ordre pour des élites post-staliniennes ébranlées par les émeutes du 17 juin 1953. Pour autant, l'absence du chef de la VP au sein du Politburo signait l'irrémédiable caractère second de cette police: tout en haut, couronnant l'immense 
système de surveillance, la Stasi (Staatssicherheit) et son omnipotent directeur limitaient toute velléité d'indépendance de la VP.

3 Les deux chapitres suivants détaillent le mode interne d'organisation de cette police si mal circonscrite (chap. 5), ainsi que la sociologie de ses personnels (chap. 6), sociologie dynamique (évolution des personnels de 1945 à 1968), socio-politique (caractéristiques sociales, générationnelles et politiques) et professionnelle (statuts et carrières). L'extraordinaire homogénéité sociale de ce corps appelé à être la " police populaire au service du peuple» se manifestait dans l'ascendance ouvrière de plus de quatre cinquièmes des Vopos. Après le 17 Juin, le Parti prit même la résolution d'interdire l'entrée des enseignants ou étudiants dans la police, privant ainsi la base de l'organisation policière d'un apport en personnels dotés de capitaux scolaires élevés ( $\mathrm{p}$. 225-226, 250-260). Cette formidable homogénéité sociale n'eut cependant pas les effets escomptés : la hiérarchie peinait à maintenir une discipline solide parmi les agents, toujours menacée par les très fortes disparités de traitement selon les grades. Finalement, mieux que toute l'économie des récompenses et gratifications, notamment dispensées au Jour de la Volkspolizei (chaque $1^{\text {er }}$ juillet de l'année), ce furent le Mur en 1961 et la fermeture de l'option « exit » qui furent dans ce combat les meilleurs alliés des cadres contre les agents du rang.

Quelles qu'en fussent les causes, la résistance particulière des agents à la discipline (que mesuraient également, dans un autre ordre d'idées, de forts taux de suicide et de tentatives de suicide, ainsi qu'une grande instabilité des personnels et un turn-over de $10 \%$ annuel de la profession ${ }^{1}$ ), est un bon indicateur de la confiance limitée des Vopos envers leur institution. Dans la Volkspolizei, contester le supérieur hiérarchique, doté de compétences policières inversement proportionnelles à son allégeance au Parti, était en fait le moyen le plus aisé de s'adresser au Parti. Est-ce à dire que les 130000 Vopos n'avaient qu'une confiance limitée dans l'État et l'idéologie qu'ils servaient ? Sans doute pas : ce qui frappe à la lecture des rapports disciplinaires des polices locales et des analyses menées par les directions (parfois au moyen de sondages de grande ampleur auprès des gradés...), ce sont les omniprésentes revendications catégorielles, salariales, sociales (une crèche pour les bébés, et un métier pour les enfants de policiers); aspirations unanimes au confort qui formaient alors le socle sur lequel le régime asseyait sa domination sur la société, domination faite d'un singulier mélange d'autoritarisme collectiviste et d'une mansuétude jamais démentie à l'égard de qui revendique son droit inaliénable à la tranquillité individuelle.

Cette rencontre des aspirations fondamentales et du champ des rétributions possibles suffit-il à expliquer que l'institution ait tenu, malgré l'étroitesse de l'horizon professionnel et l'impossible formation d'une quelconque autonomie légitimante ? Sans doute, comme le savait le gouvernement, qui introduisit en 1964 des circuits d'ascension professionnelle et de formation continue, ainsi qu'un régime social plus avantageux. L'acquisition de savoirs policiers n'était toutefois jamais distinct de l'inculcation des compétences politiques : «Si l'enseignement de ces riches savoir-faire policiers ne s'accompagne pas, dans le même temps, d'une amélioration du travail idéologique, on réduit alors de moitié la valeur de toute l'expérience que l'on pourra en tirer ». Lorsque l'État concédait un peu d'autonomie, le Parti renchérissait les moyens de contrôle : les changements introduits dans les années soixante, couronnés par la loi de 1968 sur la Volkspolizei, s'accompagnèrent d'un embrigadement renforcé de l'institution et de ses agents, dans les infinis réseaux d'obligations individuelles et de 
célébrations collectives qui constituaient cette société "imprégnée par le pouvoir » (" durchherrschte Gesellschaft ») qu'était la société est-allemande.

6 Ainsi, renforçant du même coup les logiques d'allégeance militariste, des cérémonies de serment furent introduites en même temps que le contrat de travail; lequel reconnaissait pour la première fois des «droits » au Vopo, à côté de ses classiques «devoirs ». Plus généralement, de la crèche à l'hospice, tout devenait prétexte à cérémonies, célébrations, libations, et prise en charge "totale» des existences individuelles par la Volkspolizei ${ }^{2}$. Les agents payaient ainsi les très vagues affermissements de leurs domaine de compétences (l'élaboration tâtonnante d'un «savoir» de la sécurité publique au cours des années soixante-pp. 250-260) et l'amélioration de leurs statuts par un "enrôlement » (Einbindung) de tous les instants dans une institution appelée à devenir leur seul «monde vécu», monde parfaitement refermé sur lui-même. Plus que cette police toujours fragile et incertaine, la Volkspolizei devenait une société; la société pour tous les Volkspolizisten dans un régime politique qui combattait par la formation de mille sphères dispersées toute émergence d'un espace public propre dans lequel la société tout entière pourrait opposer à l'État ses propres aspirations.

7 Comment, sur de tels fondements, se déploie le travail policier, ce que l'on appellerait ailleurs le «policing»? Thomas Lindenberger revient d'abord sur l'indifférenciation du métier policier, sur les multiples acteurs, de l'entreprise aux sociétés de loisir, avec lequel le policier est constamment requis de travailler (chap. 7). Il revient dans le détail sur les mille petites mains qui prêtaient leur concours aux policiers, des moins institutionnalisées comme les concierges d'immeubles et " relais de quartier », aux plus officiels comme les 100000 "policiers bénévoles"(freiwillige Helfer der Volkspolizei), créés en 1952, à l'égard desquels l'État prodigua également cette habile politique mettant en permanence en scène la reconnaissance enchantée de cette institution bénévole et lui refusant systématiquement toute délégation des compétences de police. Politique bifide, qui tirait avantage de son indétermination même : elle encourageait également ces policiers du dimanche à la confusion constante des tâches « techniques " et des missions « politiques ». D'une légitimité trop fragile, le Parti ne pouvait comme le régime nazi s'appuyer sur un réseau d'informateurs ou de dénonciateurs bénévoles. À défaut, il favorisait tous ces petits métiers, ces petits mondes sociaux qui lui permettaient la surveillance permanente de tous par tous et assuraient ainsi l'immense collecte des faits et pensées du petit peuple; mouvement que Lindenberger qualifie «d'étatisation permanente des relations sociales» (pp. 292-294), ou ce que Jay Rowell appellerait le patient «travail de mise en forme de la société opéré par le pouvoir étatique $»^{3}$.

8 Cette place du policier dans la société «imprégnée de pouvoir » est magistralement illustrée dans le $8^{e}$ chapitre de l'ouvrage, qui décrit le « policier de proximité » dans les campagnes. Héritière en droite ligne de la police prussienne, la Volkspolizei a su créer un dense réseau de policiers de proximité (les $\mathrm{ABV})^{4}$, de "très forte proximité ", dirions-nous, exemplaires de cette économie politique des transactions et des obligations sociales au service du pouvoir. Au pied de quelques tours ou sur une commune rurale, ces $\mathrm{ABV}$ étaient toujours disponibles à la population. Ils disposaient toujours du formulaire utile, d'un téléphone ou d'une voiture pour dépanner. À force de proximité, ils savaient tout de la population ${ }^{5}$. Au cœur de la population et si proches du pouvoir, les ABV se trouvaient dans une position avantageuse lorsque l'humeur sociale 
était apaisée, mais beaucoup plus torturée lorsqu'ils se trouvaient aux avant-postes de la politique de collectivisation et se voyaient ainsi à la fois notaires auprès de leur hiérarchie des protestations collectives, et prestataires de la bonne parole politique auprès des paysans anti-communistes, socialisés à la politique auprès des gros propriétaires prussiens. Loyautés déchirées, prises entre Parti et population. Lindenberger montre comment les ABV, dans un jeu subtil de transport calculé des protestations contre la politique de collectivisation, parviennent à se faire les discrets porte-parole des propriétaires fonciers, indispensables porte-parole, en réalité, puisqu'à force d'apprentissage ils en viennent aussi à devenir les experts agricoles des cadres politiques du Parti. Une fois les relations sociales redistribuées et stabilisées dans les campagnes, les $\mathrm{ABV}$ récoltent avec succès les profits de leur statut notabiliaire acquis au fil des ans ${ }^{6}$.

On peut imputer le succès de cette construction de rôle à l'habileté propre des ABV, beaucoup plus dotés en capitaux politiques que les fantassins de la VP. En réalité, il faut trouver dans l'ordre social des campagnes les conditions de félicité de la prise de rôle des ABV. La prise en charge collective des tâches de contrôle social et de surveillance des déviances, comme le commande partout l'ordre rural, réussissait aux ABV qui se glissaient au mieux dans les attentes d'ordre exprimées par le corps social. Lindenberger souligne ainsi que l'ordre socialiste ne réussit jamais mieux que dans les contextes sociaux où le conservatisme dominant assure à la fois l'hostilité frontale au communisme et le respect silencieux de l'ordre public. Dans les villes, en revanche, c'était une tout autre histoire qu'écrivaient le régime et ses instances de contrôle social. Blousons noirs en France, « demi-durs » en RFA (Halbstärke), « Rowdys » en RDA : le terme est inventé en 1956, dans un texte de synthèse de la VP sur les « méthodes (...) par lesquelles l'ennemi de classe sème le désordre dans la population» - p. 368). Le chapitre 9 est consacré à la prise en charge de ces Rowdys par le pouvoir, et aux luttes pour la définition légitime de l'ordre et du désordre, dans un univers balisé par la réponse militaro-policière répressive, la réponse par la mobilisation politique des masses, et la réponse par le droit de la jeunesse en danger/jeunesse dangereuse.

Les conflits furent rudes, au sommet de l'État, pour contrer ce qui était alors la seule forme un tant soit peu visible de désordre politique (du moins après le 17 juin). Lorsqu'une bande de jeunes éméchés dérouillait un soldat de l'Armée populaire sur un marché de Noël, la qualification pénale oscillait entre l'incrimination commune et la caractérisation d'un "acte rowdy " (der Rowdytum) attentatoire à la sûreté de l'État. Lindenberger engage alors rien moins qu'une recherche sur la genèse de la délinquance juvénile et sa construction comme problème social par le Parti et l'État, qui éclaire d'une lumière neuve les débats de nos démocraties contemporaines. Au final, note-t-il, il est tout à fait exemplaire que la solution de traitement de la délinquance par les masses, et notamment par l'organisation de jeunesse FDJ, échoua : le problème résistait dans sa spécificité et appelait une force répressive professionnelle. C'est ainsi que l'on vit au fil des années soixante se constituer, pour la première fois, un savoir criminologique spécifique autour de la déviance et de ses frontières, fixant peu à peu la notion de " délinquance » (et non plus de " déviance politique ») dans l'horizon cognitif des juristes et plus largement des cercles dirigeants du Parti. La solution choisie, à la fin des années soixante, fut celle d'un renforcement du contrôle social non pas des jeunes, mais depuis les jeunes : mieux surveiller les parents, mieux encadrer les enseignants, et leur déléguer le contrôle des enfants. À côté de cela, un savoir particulier de «stérilisation" des espaces publics se forgeait, visant à tuer dans l'œuf tout 
rassemblement de jeunes. La "prévention » made in DDR était alors à la fois délégation du contrôle social aux autorités classiques (famille, école) et généralisation des mesures préventives de privation temporaire de liberté en cas d'événements publics. Contrôle traditionnel, police rudimentaire.

De cet immense travail il faut d'abord retenir les jeux permanents de décentrement du regard historien qui se porte ici sur les processus d'élaboration des normes, là sur l'application de ces normes; ici sur la composition sociologique de l'institution globale, là sur les conflits disciplinaires locaux; ici sur les conflits au sein des instances doctrinaires ou partisanes autour de la délinquance juvénile, là sur les rapports policiers de tel arrondissement sur la prise en charge concrète du phénomène. C'est toute la capillarité du pouvoir qui est dévoilée par l'étude de la Volkspolizei au quotidien, cette police qui comme nombre d'institutions qui grandirent sur le sol estallemand incarnait le Parti mais, dans le même temps, se trouvait enracinée dans les moindres linéaments de la vie quotidienne de la société.

Lindenberger dégage ainsi une caractéristique forte de la domination «réelsocialiste " : l'incessante invention de groupes sociaux et de corporations par le Parti, la consécration de ces corps en mondes sociaux totaux, et la perpétuelle défaite de toute revendication à l'autonomie de ces mondes. Si le régime totalitaire oppose l'omnipotence de l'État à la nudité de l'individu, le régime est-allemand étouffait quant à lui l'individu dans ces mille micro-sociétés que le Parti suscitait et ne cessait d'animer, de mobiliser, de bouleverser ou de célébrer, pour mieux les étouffer.

Quelle fut la place réelle, dans ce patient édifice de domination bifide, de la police du quotidien? Une place assurée par l'histoire, en premier lieu, celle que la police a toujours occupée jusque l'avènement du nazisme. La Volkspolizei reprend à son compte l'héritage weimeiro-prussien que l'historien Herbert Reinke caractérisait comme "eudémonisme patriarcal aux couleurs policières» et que Thomas Lindenberger dépose sur le compte aujourd'hui clos de la Volkspolizei. Mieux que tout autre en effet la Volkspolizei de la République démocratique allemande porte l'héritage des polices conservatrices et patriarcales de l'Allemagne du XIX ${ }^{e}$ siècle et de Weimar, interrompues par la catastrophe nazie, qui développa avec une police de la terreur une tout autre institution. Mais Lindenberger, en cela fidèle à l'école de l'histoire sociale du quotidien, la Alltagsgeschichte ${ }^{7}$, ne veut jamais trop croire à la force seule des institutions : il montre combien la stabilité gagnée envers et contre tout par cette police si précaire devait à la filiation de la société est-allemande avec les sociétés prussiennes : la « longue durée (en français dans le texte) des habitudes ancrées de comportements et d'attentes", également parmi les groupes ouvriers, explique la stabilité des représentations collectives de l'ordre et du désordre (p. 457). Cet État aux aspirations totalitaires était à la fois enclin à offrir tous les moyens à sa police d'assurer sa pérennité, et pouvait se reposer sur le conservatisme hérité de la longue durée pour la priver toujours du monopole de la contrainte légitime. Sous la plume de Thomas Lindenberger, et même s'il ne présente pas son travail ainsi (ou peut-être le ferait-il s'il développait un peu plus la notion de gouvernementalité qu'il convoque parfois), l'histoire sociale, sans quitter l'histoire des pratiques sociales, devient aussi une histoire sociale de l'État lui-même. Non contente de seulement documenter la distance entre les 
pratiques sociales constatées et les normes imposées par l'État, l'histoire sociale de la police et de ses conditions quotidiennes de réalisation devient une histoire de l'État pris dans le social, qui dévoile tout ce que l'État doit aux formes sociales dont il procède et contre lesquelles il se débat pour s'imposer.

\section{NOTES}

1. Un renouvellement annuel de $10 \%$ des agents correspond ainsi, en données cumulées, à un total de 90000 départs de 1955 à 1963, pour un corps de 130000 agents (p. 212); ce qui correspond sans doute à une des plus fortes fluctuations sur une période aussi longue parmi les corps policiers européens (pour comparaison, la police « réunifiée » s'est séparée à Berlin Est d'environ la moitié de ses membres seulement, soit le taux largement le plus élevé de turn-over de tous les Länder de l'est après 1990, mais sur une durée d'une à deux années; l'instabilité constante et continue de la Volkspolizei est à cet égard remarquable).

2. Sur ces espaces publics organisés, voir l'étude de Sandrine Kott, Le communisme au quotidien. Les entreprises d'État dans la société est-allemande, Paris, Belin, coll. « socio-histoire », 2001.

3. Jay Rowell, «L'histoire sociale de l'État RDA vue à travers le prisme de la politique du logement », Berlin, Centre Marc Bloch, Working Paper n 6, juin 2002, p. 4 (disponible en ligne : www.cmb.hu-berlin.de).

4. Littéralement «plénipotentiaires de circonscription» (Abschnittsbevollmächtigte), dotés de moyens et de compétences à la mesure de leur importance pour le pouvoir.

5. On n'insistera pas plus longuement sur cette ironique double apparence des programmes de « community policing " ou « neighbourhood watch »: les importations anglo-saxonnes très en vogue en Allemagne depuis la fin des années 1980 sont souvent perçues à l'Est sous le jour de l'héritage voire de la restauration (Lindenberger le souligne pp. 458-460).

6. On trouvera ce processus développé avec brio dans Thomas Lindenberger, «La police populaire de la RDA de 1952 à 1958. Une micro-étude sur la gouvernementalité de l'État socialiste ", Annales HSS, 53, 1, 1998, pp. 119-152.

7. Voir l'ouvrage dirigé par Alf Lüdtke: The history of everydaylife. Reconstructing historical experiences and ways of life, Princeton, 1995 et, sur l'apport de ces avancées historiographiques à l'analyse de la RDA, du même auteur, «La RDA comme histoire. Réflexions historiographiques ", Annales EHS, 53, 1, 1998, p. 33 et suiv.

\section{AUTEURS}

\section{FABIEN JOBARD}

Cesdip-CNRS (Guyancourt, France), fabjob@cesdip.com 